\title{
System Design Considerations for Undersea Networks: Link and Multiple Access Protocols
}

\author{
Nathan Parrish, Student Member, IEEE, Leonard Tracy, Student Member, IEEE, Sumit Roy, Fellow, IEEE Payman \\ Arabshahi, Senior Member, IEEE, and Warren L. J. Fox, Senior Member, IEEE
}

\begin{abstract}
We address several inter-related aspects of underwater network design within the context of a cross-layer approach. We first highlight the impact of key characteristics of the acoustic propagation medium on the choice of link layer parameters; in turn, the consequences of these choices on design of a suitable MAC protocol and its performance are investigated.

Specifically, the paper makes contributions on the following fronts: a) Based on accepted acoustic channel models, the pointto-point (link) capacity is numerically calculated, quantifying sensitivities to factors such as the sound speed profile, power spectral density of the (colored) additive background noise and the impact of boundary (surface) conditions for the acoustic channel; b) It provides an analysis of the Micromodem-like linklayer based on FH-FSK modulation; and finally c) it undertakes performance evaluation of a simple MAC protocol based on ALOHA with Random Backoff, that is shown to be particularly suitable for small underwater networks.
\end{abstract}

Index Terms-Underwater acoustic communications, Information rate, Access protocols.

\section{INTRODUCTION}

$\mathbf{N}$ ETWORKED observatories comprised of fixed and mobile underwater (UW) nodes are being conceived and increasingly deployed for many different environmental monitoring scenarios. Of particular significance is the role of monitoring the physical, chemical and biological properties of the ocean system as a critical component of the overall grand challenge problem of climate prediction.

Broadly speaking, there exists a great need for continual, real time monitoring of the ocean's properties based on dense spatio-temporal sampling. Since as much as $90 \%$ of the ocean volume is unexplored, several regional undersea observatories have been recently deployed in partial amelioration of this deficit. One example (pertinent to the Pacific NorthWest) is the NEPTUNE project [1] that consists of an initial cabled sea-bed infrastructure primarily intended for monitoring of sea-floor events. It is expected that this will expand in time to include vertical moored profilers as well as underwater

Manuscript received February 15, 2008; revised September 2, 2008. This work was supported by the NASA Earth Science Technology Office's Advanced Information Systems Technology (AIST) Program under award number AIST-05-0030.

N. Parrish, L. Tracy, and S. Roy are with the Dept. of Electrical Engineering, University of Washington, Box 352500, Seattle, WA 98195 (e-mail: nparrish,lentracy,sroy@u.washington.edu).

W.L.J. Fox is with BlueView Technologies, 2151 N. Northlake Way, Suite 101, Seattle, WA 98103 (e-mail: warren.fox@blueviewtech.com).

Payman Arabshahi is with the Applied Physics Laboratory and the Dept. of Electrical Engineering, University of Washington, Box 355640, Seattle, WA 98195, (e-mail: payman@ee.washington.edu).

Digital Object Identifier 10.1109/JSAC.2008.081211. autonomous vehicles (UAVs). Another semi-autonomous network - PLUSNET - consisting of fixed bottom and mobile sensors is oriented toward surveillance applications such as tracking ships and submarines operating in shallow water environments typical of the Western Pacific [2].

Real-time observation of the ocean requires reliable acoustic communication between both fixed and mobile underwater nodes. The objective of this paper is to provide an integrated system design approach to such UW acoustic networks, by highlighting the interplay between the acoustic medium and its consequent impact on design choices at the link and multiple access (MAC) layers; see [3] for a recent review of UW network design issues.

Fundamental to UW network performance analysis is the choice of suitable acoustic channel models - a research area of considerable sophistication that has yielded detailed, numerical computation-intensive models for the received acoustic field as a function of the propagation environment. However, our cross-layer approach necessitates a balance between model accuracy and computational complexity, as the latter largely determines the feasibility of simulation-based network-level performance analysis. Accordingly, in our work we have opted for simpler models that nonetheless capture the important gross features of the acoustic medium; the intent is to provide sensitivity analysis as a function of the key environmental parameters. Pushing the cross-layer agenda inherently induces such compromises, whereby the loss of accuracy in channel modeling is hopefully compensated by the enhanced insights made available into link and MAC layer design choices.

The insights from the link level analysis coupled with the intended network scenarios and consideration of various system constraints leads to design choice of a simple MAC protocol based on ALOHA with random backoff. Evaluation of the MAC performance is conducted using the freeware simulation environment ns-2, that quantifies the improvement in channel utilization relative to pure ALOHA. We note that the link-optimized MAC layer proposed here has been implemented in the micro-modem, and will be undergoing sea trials in the near future. Aside from the US Navy Seaweb project $^{1}$, our work is one of few open-source, field validated implementations of an underwater network.

\footnotetext{
${ }^{1}$ The Seaweb project seeks to enable undersea sensor networks, autonomous/unmanned underwater vehicle (AUV/UUV) communication and navigation, and submarine communications at speed and depth (CSD). Using an aggressive experimental program based on a proprietary version of the Benthos ATM family of modems, Seaweb seeks to determine quality of service (QoS) trade-offs of underwater networks in terms of reliability, availability, throughput, area coverage, security, and latency.
} 


\section{A. UW Network Engineering Challenges}

As the demands on underwater data communication rates increase, so does the need for understanding the limits of transport of the acoustic medium. Traditionally, the Shannon capacity (or maximum achievable rate with arbitrarily low error probability) of any channel perturbed by additive Gaussian noise (AGN) has served as the benchmark for point-topoint communications. Nonetheless, there have been only a few recent attempts to compute and characterize such link capacities over underwater AGN channels. Part of this is attributable to the fact that channel modeling for undersea acoustics is still an ongoing art due to its many additional complexities as compared to terrestrial scenarios.

By way of an example, the boundary conditions for a shallow body of water, i.e. the surface and bottom profiles, exert considerable impact via energy loss and scattering of acoustic waves, and thus on the link capacity. In terrestrial communications, typical propagation models between two fixed nodes such as the two-ray model provide a relation for signal power attenuation as a function of transmitter - receiver separation that is independent of frequency. Therefore, given a pair of nodes communicating through an additive white Gaussian noise (AWGN) channel at some fixed transmit power and bandwidth, the capacity is only a function of distance. However, in underwater communications, both the attenuation and noise are known to be strong functions of frequency. See for example, the recent computations in [4], [5] that explicitly recognize the frequency selective nature of acoustic attenuation and noise.

Additionally, acoustic propagation is characterized by the sound speed profile, which can vary greatly even in shallow water. The sound speed profile can show great variability at different times of year and under different weather conditions. Typical non-uniform sound speeds with respect to water depth cause a 'bending' of acoustic pressure waves.

We will show that the channel sound speed profile and boundary characteristics play a large role in determining the capacity between a source and receiver pair. Additionally, two different receivers at the same distance from a transmitter have very diverse capacities depending on their vertical location relative to the transmitter and the sound speed profile. In our work, we undertake an analysis of such parametric sensitivities of the channel capacity to highlight the unique features of UW networks.

We also conduct a link performance analysis of the Woods Hole Oceanographic Institution's (WHOI) Micromodem [6]. The primary reason for doing so is that the Micromodem remains the only acoustic modem available to the community at large as a platform for research and integration into UW networks. While there exist other commercially available modems (notably by Teledyne Benthos) and there are ongoing efforts to develop new software defined acoustic modems, none of them offer the support and flexibility of the WHOI Micromodem ${ }^{2}$.

${ }^{2} \mathrm{~A}$ Communication stack application programming interface (API) for the Micromodem was developed at the University of Washington's Applied Physics Laboratory for this purpose, i.e. for implementing the MAC layer described in this work.
We next incorporate the results of our link layer analysis into a proposed MAC protocol that is suited to channels with long propagation delays. Our design philosophy is driven by pragmatism and seeks to avoid the complexities of network timing synchronization while pursuing enhanced channel utilization; this implies the need for simple collision avoidance mechanisms, leading to the choice of ALOHA with a random back-off. The value of our work in this regard is two-fold:

i) The proposed simple MAC protocol is consistent with existing UW acoustic modem hardware, such as the WHOI Micromodem and can be readily implemented for field evaluations. It can also be used with little or no modification in other modems such as the one currently being developed by Teledyne Benthos that will include both the WHOI and Benthos link layers. ii) In terms of MAC performance analysis (conducted with the popular freeware network simulator ns-2), a suitable abstraction is used for link losses within the protocol simulation. To the best of our knowledge, this represents one of the first cross-layer attempts for UW network evaluation within an open-source simulation environment.

The rest of the paper is organized as follows. Section II gives an overview of Bellhop and its use to model the underwater acoustic channel. Section II also includes analysis for underwater channel capacity using a frequency selective AGN model for three channels exhibiting varying sound speed profiles. Section III performs a link analysis for an FH-FSK modem using a Bellhop channel model. Section IV proposes a MAC protocol for a network of underwater communications nodes using the Woods Hole Micromodem. The protocol is analyzed under one of the same Bellhop Channel profiles given in Section II. Section V concludes the paper.

\section{Point-To-Point Link CAPACity}

\section{A. Acoustic Modeling Using Bellhop}

In modeling underwater acoustic propagation for acoustic telemetry, we are interested in methods that achieve a good balance between accuracy in representation of the key features with acceptable run times. In the realm of high frequency acoustic modeling, numerical approaches based on Gaussian ray tracing provide such a compromise. We adopt Bellhop (developed by M. Porter) for our work; descriptions of the method and the code can be found at [7] and [8], respectively. Bellhop's Gaussian ray tracing generates a complex channel impulse response for any user defined input channel conditions, that can be used for subsequent link layer performance analysis.

The relevant environmental conditions that influence the channel impulse response include the channel depth, sound speed profile (SSP), surface and bottom conditions, and the frequency bands in use. The SSP influences the propagation of acoustic rays by 'bending' them according to Snell's law. Interface conditions and the frequency of transmission both influence losses of acoustic energy. Although conservation of energy holds, energy in the form of acoustic pressure within the medium is lost due to two processes: 1) transmission out of the water medium and into the sediment at the bottom interface and 2) frequency dependent dissipation as heat. We model both the top and bottom surfaces as homogeneous liquid 
halfspaces. Under this assumption, losses of acoustical energy occur based on the transmission and reflection coefficients. When rays are incident with the bottom surface, the fraction of the ray's energy transmitted into the soil is determined by the specific acoustical properties of the bottom material as well as the grazing angle of the incident ray. Bellhop allows the user to enter the acoustical properties of the bottom surface in order to model this loss. Additionally, acoustic energy is dissipated as heat due to frequency selective Thorp attenuation, given as

$$
\begin{gathered}
A(d, f)=d \cdot 10 \log (a(f)) \\
10 \log (a(f))=0.1 \frac{f^{2}}{1+f^{2}}+40 \frac{f^{2}}{4100+f^{2}}
\end{gathered}
$$

where $10 \log (a(f))$ is Thorp Attenuation, given in $\mathrm{dB}$ per kiloyard. $A(d, f)$ is the absorption loss in $\mathrm{dB}$ for a ray at frequency $f$, in $\mathrm{kHz}$, which travels $d$ kiloyards.

Scattering at rough interfaces represents the loss of beam coherency. The Rayleigh parameter, $R$, given in [9] as $R=$ $2 k H \sin (\alpha)$ can be used to roughly determine if a ray is reflected coherently $(R \ll 1)$ or is scattered $(R \gg 1)$ based on the wavenumber $(k)$, the RMS surface displacement $(H)$, and the grazing angle $(\alpha)$. A simple model for analyzing surface scattering is based on the coherent beam losses. Urick [9] defines the amplitude reflection coefficient as the ratio of coherent reflected amplitude to the incident amplitude $u=e^{-R}$. We determine the RMS wave height in meters, $H$, assuming a Pierson-Moskowitz model for the sea state as $H=5.33 \times 10^{-3} w^{2}$ where $w$ is the wind speed in meters per second [10]. We use this simple model to represent losses at the sea surface due to roughness.

\section{B. Link Capacity}

The received bandpass signal in an underwater acoustic channel may be generally written as

$$
r(t)=\sum_{l=1}^{L} \operatorname{Re}\left[h_{l}(t) e^{\left(j 2 \pi f_{c}\left(t-\tau_{l}\right)\right)} x\left(t-\tau_{l}\right)\right]+n(t)
$$

which represents the superposition of $L$ copies of the transmitted lowpass (narrowband) signal $x(t)$ modulated at carrier frequency $f_{c}$ arriving at the receiver, with respective delays $\tau_{1}, \ldots, \tau_{L}$. The values $h_{1}(t), \ldots, h_{l}(t)$ are the complex gains introduced by the channel. Without loss of generality, we assume that the first arrival occurs at delay $\tau_{1}=0$. If the symbol period is less than the maximum delay $\tau_{l}$, then the channel is frequency selective, leading to inter-symbol interference (ISI). On the other hand, if the symbol period is significantly greater than $\tau_{l}$, there is no ISI, and the received signal becomes:

$$
\begin{aligned}
& r(t)=\operatorname{Re}\left[\alpha(t) x(t) e^{\left(j 2 \pi f_{c} t\right)}\right]+n(t) \\
& \alpha(t)=\sum_{l=1}^{L} h_{l}(t) e^{-j 2 \pi f_{c} \tau_{l}}
\end{aligned}
$$

Due to low symbol rates, we assume that Eq. (4) models the received signal. However, despite the lack of ISI, we note that acoustic attenuation and ambient sea noise characteristics induce significant frequency dependence.
In order to compute the channel capacity we make the following assumptions. We assume that the channel impulse response is constant over the period of observation. We also assume that the ambient noise term, $n(t)$ is additive colored Gaussian with a p.s.d determined by the various components (wind, thermal, turbulence, and shipping) given in [11]. Under these assumptions, we can compute the channel capacity by the usual approach: the signal bandwidth is divided into sufficiently narrow (ie. frequency non-selective), equi-spaced frequency bins, and the net transmit power $P$ is allocated among the bins via 'water filling'. The capacity of each bin is then computed and summed to obtain the aggregate capacity. The bins are sufficiently small such that the channel impulse response, frequency selective attenuation, and noise can be assumed to be constant across the bin. For this to be true, the bandwidth of each bin must be the smaller of $1 / \tau_{l}$ or 100 $\mathrm{Hz}$. The first requirement ensures that the symbol period is larger than the delay spread of the channel while the second ensures that the frequency selective attenuation and noise can be assumed constant across the frequency bin. Under these assumptions, the received signal in the $n$-th bin is given as:

$$
\begin{aligned}
& r_{n}(t)=\operatorname{Re}\left[\alpha_{n} \sqrt{P_{n}} x(t) e^{\left(j 2 \pi f_{n} t\right)}\right]+n_{n}(t) \\
& \alpha_{n}(t)=\sum_{l=1}^{L} \operatorname{Re}\left[h_{n, l}(t) e^{-j 2 \pi f_{n} \tau_{l}}\right]
\end{aligned}
$$

where $f_{n}$ is the center frequency of the $n$-th bin and $P_{n}$ is the corresponding power allocation.

The channel capacity is therefore given by [12]:

$$
C(f)=\sum_{\max P_{n}: \sum P_{n} \leq P} B \log _{2}\left(1+\frac{\left|\alpha_{n}\right|^{2} P_{n}}{N_{n} B}\right) b / s
$$

where $\alpha_{n}$ is given in Eq. (5), $N_{n}$ is the noise power spectral density (p.s.d.) for the $n$-th bin, and $B$ is the bin bandwidth. We determine the noise p.s.d. from its various components given by Coates [11], which, in the frequency bands useful for underwater communications, are highly dependent on wind speed and shipping activity.

We now apply the capacity calculations defined above to several different underwater channels. We show the capacity under different bottom conditions, wind speeds, and SSPs. We assume that the bottom is composed of 'silty clay' unless otherwise mentioned, the acoustical properties of which can be found in [13]. We use a channel bandwidth of $22-26$ $\mathrm{kHz}$, and transmit power of $190 \mathrm{~dB}$ re $1 \mathrm{uPa}$. Additionally, on all plots we include the capacity of the channel based on uniform spreading and Thorp attenuation as a reference for comparison. We do this by calculating the value of $\left|\alpha_{n}\right|^{2}$ in (6) as:

$$
\begin{gathered}
10 \log \left(\left|\alpha_{n}\right|^{2}\right)=k \cdot 10 \log \left(d \cdot 10^{3}\right)+d \cdot 10 \log \left(a\left(f_{n}\right)\right) \\
10 \log \left(a\left(f_{n}\right)\right)=0.1 \frac{f_{n}^{2}}{1+f_{n}^{2}}+40 \frac{f_{n}^{2}}{4100+f_{n}^{2}}
\end{gathered}
$$

The value $f_{n}$ is the center frequency of bin $n, k$ is the spreading coefficient, and $d$ is the distance in kiloyards. Bins are separated by $100 \mathrm{~Hz}$. We use $k=1.5$ for the spreading coefficient as was done in [4] to represent a compromise between spherical and cylindrical spreading. 


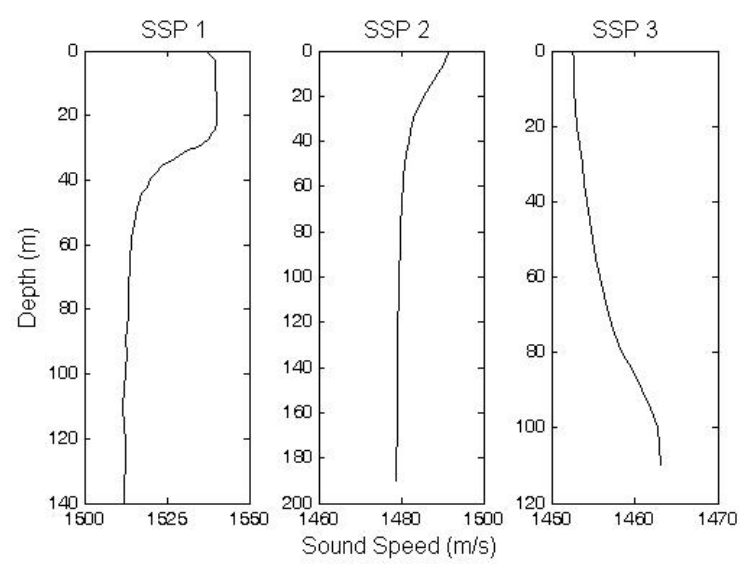

Fig. 1. Sound Speed Profiles studied for the capacity calculations in Section III

\section{Link Capacity Results}

We examine channels exhibiting three distinct sound speed profiles. The first exhibits a warm mixed layer at the surface with high sound speeds, then a thermocline where the sound speed drops drastically. The second exhibits a downward refracting sound speed profile, and the third exhibits an upward refracting sound speed profile. These three profiles are shown from left to right in Fig. 1. These SSPs were measured in the Mediterranean Sea in September, in June in Griffin Bay near the San Juan Islands, and in February in the Bering Sea respectively.

Figs. 2 - 5 give capacity calculations for different source and receiver locations within these different SSPs under varying surface and bottom conditions. We can see from these figures that the specific channel variabilities significantly impact the channel capacity. We can compare Figs. 2(a) and 3 to see the influence of bottom type. Bottom losses due to 'medium sand' are less than those due to 'silty clay', and we see this effect clearly in the estimated capacity. From Fig. 2 when the source and receiver are both located either above or below the thermocline, the capacity is greater than when they are on opposite sides. Additionally, surface losses due to wind impact the source and receiver above the thermocline much more than the source and receiver below the thermocline. Fig. 5 shows that surface losses due to increased wind noise play a significant role at all source and receiver locations when the SSP is upward refracting. While these results conform to general intuition, they provide numerical estimates of the impact of these important channel parameters vis-a-vis Thorp attenuation and uniform spreading and provide guidance for node placements in an underwater network.

\section{Link Performance in Acoustic Channels: FREQUENCY HOPPED-FREQUENCY SHIFT KEYING} (FH-FSK)

Achieving rates close to capacity on the underwater communications channel continues to be a challenge. For example, in our capacity calculations, we neglected any ISI and assumed that the receiver captures all of the transmitted signal power. Since underwater channels typically exhibit significant delay spreads, this is unlikely to be the case. Hence, limitations

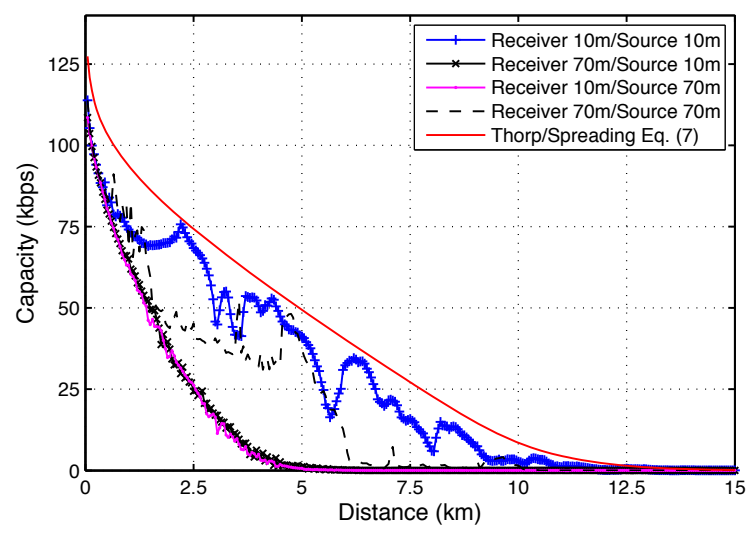

(a)

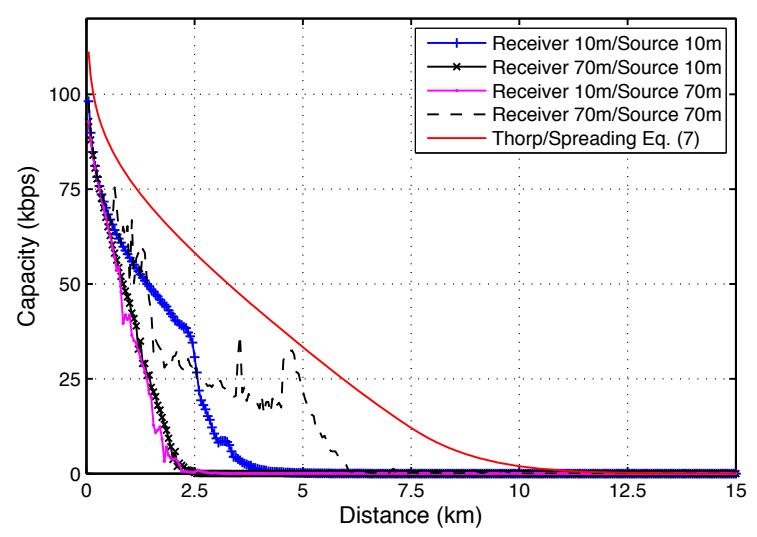

(b)

Fig. 2. Capacity for SSP 1 in Fig. 1 with a 'silty clay' bottom and wind speed of $2.5 \mathrm{~m} / \mathrm{s}$ (a) and $10 \mathrm{~m} / \mathrm{s}(\mathrm{b})$.

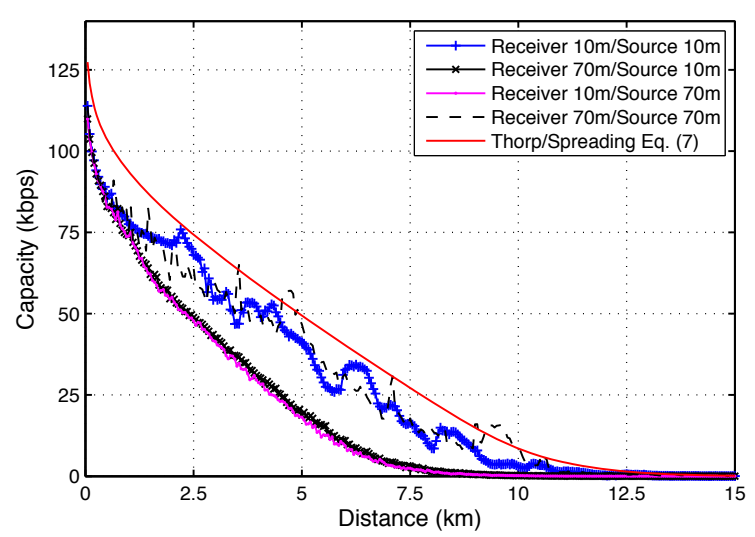

Fig. 3. Capacity for SSP 1 in Fig. 1 with a 'medium sand' bottom and wind speed of $2.5 \mathrm{~m} / \mathrm{s}$.

in terms of processing speed and energy considerations on current underwater platforms strongly impact the state-ofart in UW modem speeds. UW modems today typically use spectrally inefficient coding and modulation schemes, thereby trading off rate with robustness to channel impairments.

We now turn our attention to link performance of an $\mathrm{FH}-$ FSK modem such as the Micromodem [14] [6]. We assume that the modem transmits at a channel rate of 80 bits per sec- 


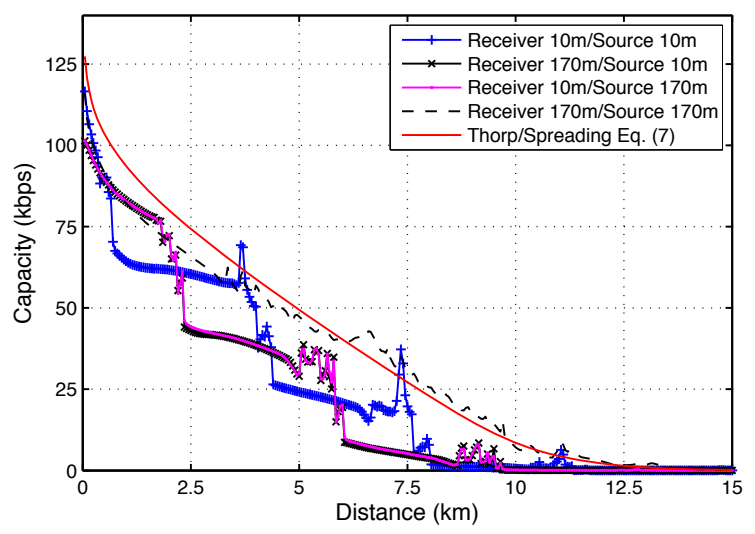

(a)

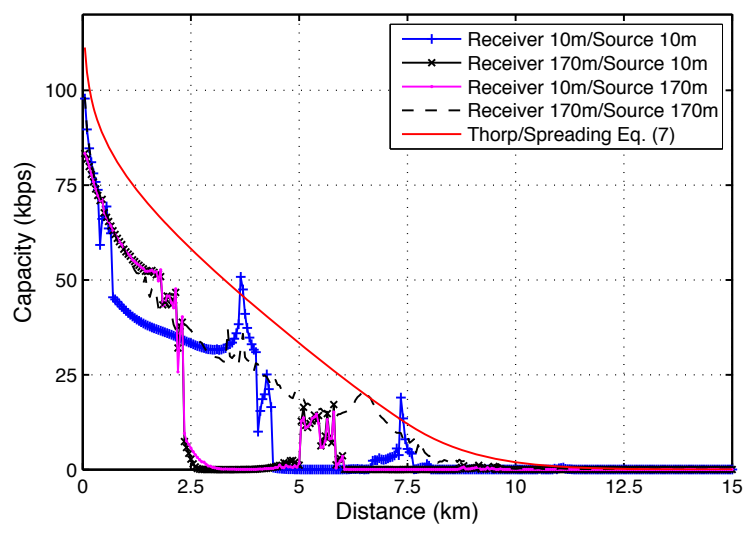

(b)

Fig. 4. Capacity for SSP 2 in Fig. 1 with a 'silty clay' bottom and wind speed of $2.5 \mathrm{~m} / \mathrm{s}$ (a) and $10 \mathrm{~m} / \mathrm{s}$ (b).

ond utilizing binary FH-FSK with 13 hopping bins as shown in Fig. 6. The modulator and demodulator hop through these bins in a pre-determined pattern and perform non-coherent binary FSK in each bin. The inter-bin frequency separation is $160 \mathrm{~Hz}$, with 2 frequencies at each bin; therefore the total system bandwidth is approximately $4 \mathrm{kHz}$. The transmitter and receiver spend $12.5 \mathrm{~ms}$ in each bin before hopping to the next one, so a complete cycle time is $150 \mathrm{~ms}$ that equals the 'clearing' time on any single narrowband channel. Since the bins are presumed orthogonal, inter-symbol interference is mitigated for channels with delay spreads less than the above channel clearing time.

We now assume a slowly, time varying channel (see Eq. (3)) represented as a discrete tap delay line with taps at intervals of the symbol period. The complex gain of the $m$-th tap is:

$$
\alpha_{m}=\sum_{l} h_{l}(t) e^{\left(-j 2 \pi f_{c} \tau_{l}\right)} \quad l \text { s.t. } m T_{s} \leq \tau_{l}<(m+1) T_{s}
$$

where $T_{s}$ is the symbol period and $h_{l}$ and $\tau_{l}$ are the gain and delay of the unresolvable arrivals within the $m$-th interval. Note that in the following derivation, the channel attenuation and noise p.s.d are assumed constant across any bin. The $k$-th

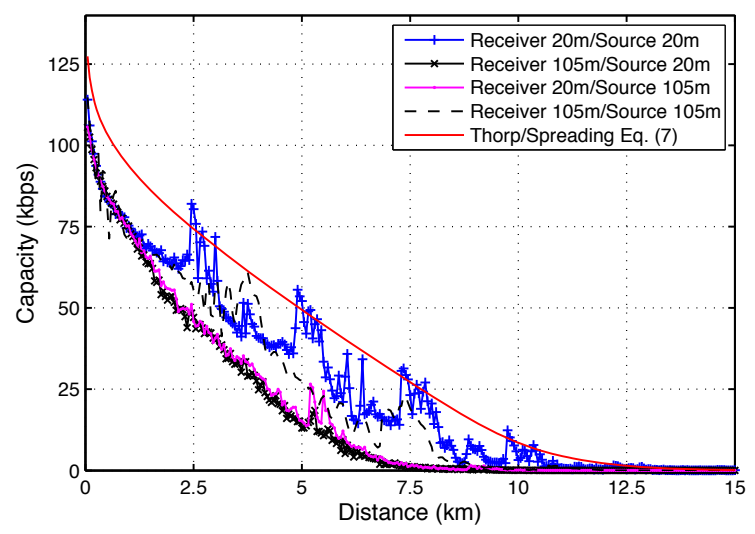

(a)

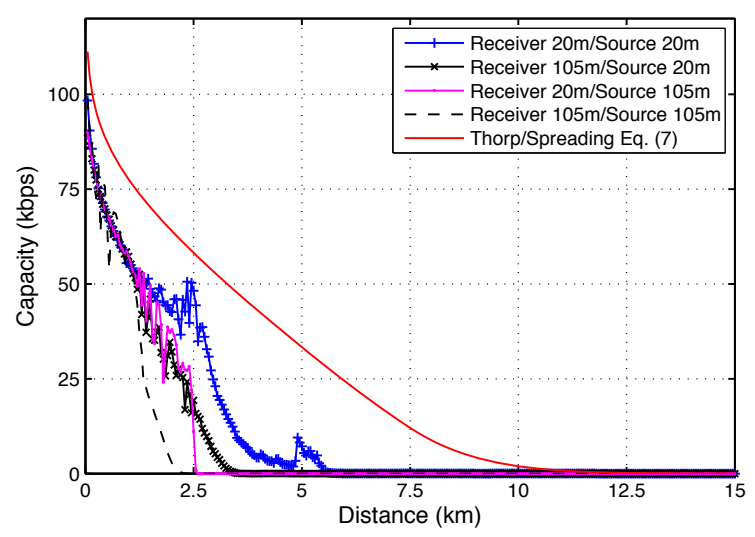

(b)

Fig. 5. Capacity for SSP 3 in Fig. 1 with a 'silty clay' bottom and wind speed of $2.5 \mathrm{~m} / \mathrm{s}$ (a) and $10 \mathrm{~m} / \mathrm{s}$ (b).

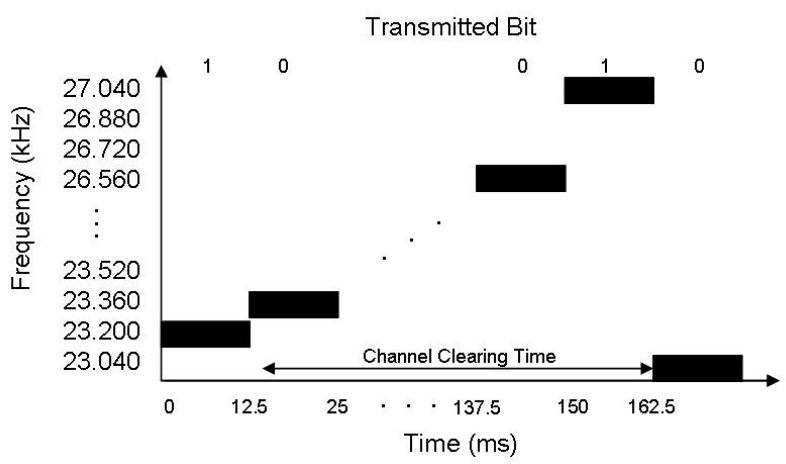

Fig. 6. Hopping Pattern for Binary FH-FSK

sample of the received lowpass signal is therefore

$$
r_{k}=\sum_{m=1}^{M} \alpha_{m} x_{k-m}+n_{k}
$$

where $x_{k}$ is the transmitted symbol sequence and $n_{k}$ is a sample of additive Gaussian noise.

It is well-known [15] that the probability of error for noncoherent binary FSK in an ISI-free fading channel can be derived by averaging the probability of the signal to noise ratio (SNR), $\gamma_{b}=|\alpha|^{2} E_{b} / N_{0}$, over the probability of error in AWGN where $E_{b}$ is the energy per bit, and the noise power 
spectral density is $N_{0} / 2$ :

$$
P_{2}=\int_{0}^{\infty} P_{e}\left(\gamma_{b}\right) P_{\gamma_{b}}\left(\gamma_{b}\right) d \gamma_{b}
$$

Therefore, the probability of error is dictated by the the distribution of $\gamma_{b}$. If we assume that the amplitude of the arrivals is Rayleigh distributed, then $P_{\gamma_{b}}\left(\gamma_{b}\right)=\frac{1}{\gamma_{b}} e^{\left(-\gamma_{b} / \overline{\gamma_{b}}\right)}$ where $\overline{\gamma_{b}}=\mathrm{E}\left[|a|^{2}\right] E_{b} / N_{0}$ and $\mathrm{E}[]$ denotes expected value. The well known result for binary FSK is:

$$
P_{2}\left(\bar{\gamma}_{b}\right)=\frac{1}{2+\bar{\gamma}_{b}}
$$

To include the effect of ISI indicated by Eq. (10), we use the expected value of the signal to multipath ratio (SMR) as opposed to the SNR in Eq. (12). The expected value of the SMR, assuming that the tap gains are independent, is given as

$$
\bar{\gamma}_{b m}=\frac{\mathrm{E}\left[\left|\alpha_{0}\right|^{2}\right] E_{b}}{\left(N_{0}+\sum_{n} \mathrm{E}\left[\left|\alpha_{D n}\right|^{2}\right] E_{b}\right)}
$$

where the second term in the denominator of Eq. (13) is the ISI energy during the sampling interval, modeled as equivalent Gaussian noise. The index for the ISI is at intervals of $D$, the number of hops in the hopping pattern. As mentioned earlier, in an FH system there is a channel clearing time that eliminates some of the ISI; therefore only the multipath arrivals that arrive at the receiver in delay intervals corresponding to an integer multiple of the clearing time $D T_{s}$ are considered as potential interference.

Siderius et al show in [16] that assuming a Rayleigh distribution with SMR as the received amplitude metric closely matches experimental data. Since we are interested in using a suitable physical layer model to drive the probability of error in a MAC layer simulator, we next adapt the received signal power to include the interference, i.e. arrivals from neighboring nodes that collide in both time and frequency with the desired signal. We also model these arrivals as additive noise, giving the (final) average SINR as

$$
\bar{\gamma}_{b i}=\frac{\mathrm{E}\left[\left|\alpha_{0}\right|^{2}\right] E_{b}}{N_{0}+\sum_{n} \mathrm{E}\left[\left|\alpha_{(D n)}\right|^{2}\right] E_{b}+\sum_{i} \mathrm{E}\left[\left|d_{i}\right|^{2}\right] E_{b}}
$$

where the final term in the denominator is the interference power from $i$ interferers with corresponding path gains $d_{i}$. The above equation indicates the need to determine the average path gains for the channels, typically called the power delay profile. We again use a Bellhop model to accomplish this for a given channel, by perturbing the SSP by a sequence of i.i.d. zero mean Gaussian random variables (one at each depth where the SSP is defined). For each SSP, we compute the squared magnitude of each tap from the channel impulse response given by Bellhop using Eq. (9), and then average to determine $E\left[\left|\alpha_{m}\right|^{2}\right]$ for each tap.

Using this method, we computed the power delay profile for channels at two depths for SSP 1 in Fig. 1 with a 'medium sand' bottom and wind speed of $0 \mathrm{~m} / \mathrm{s}$. Fig. 7(a) represents a source and receiver both at $70 \mathrm{~m}$ depth separated by 3 $\mathrm{km}$. In (b), the source is at $70 \mathrm{~m}$ depth and the receiver at $10 \mathrm{~m}$ depth, again separated by $3 \mathrm{~km}$. In these figures, we assume that our receiver will acquire timing based on the largest impulse response component, which was aligned to

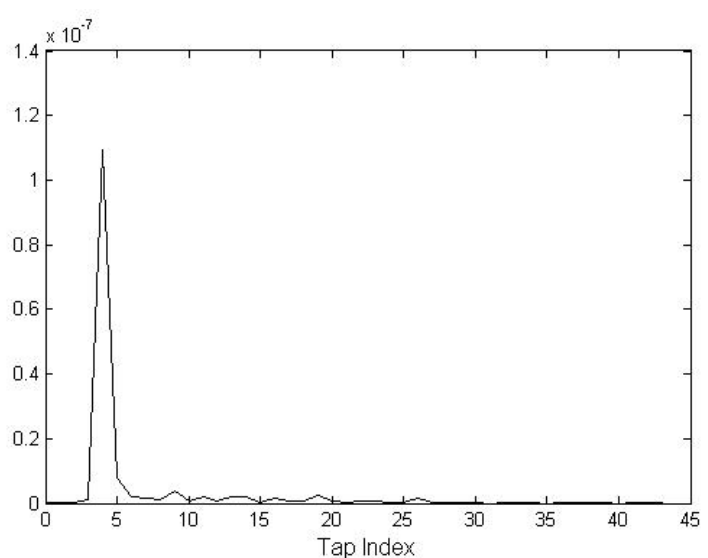

(a)

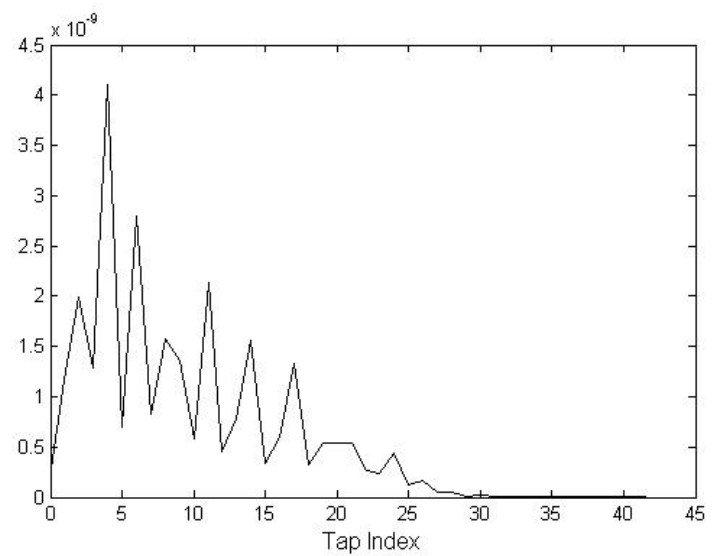

(b)

Fig. 7. Expected magnitude for two different tap delay line channels. We use SSP1 of Fig. 1 with a 'medium sand' bottom type and the distance between source and receiver is $3 \mathrm{~km}$. In (a) both source and receiver are below the thermocline. In (b) the source is below the thermocline and the receiver is above.

be the fourth tap. We see that the channel is much more favorable when the source and receiver are each below the thermocline, as the channel is dominated by a large coherent arrival. When the receiver is above the thermocline, the power delay profile indicates that the channel is ISI dominated. An analysis in [16] using experimental data to perturb the SSP gives similar results. Clearly, these two channel power delay profiles will lead to very different modem link performance. In the following section we will explore how, in turn,this affects MAC design.

In determining the final probability of error, we also include the effect of a rate $r_{c}=\frac{1}{2}$ convolutional code of constraint length 9 with soft decision Viterbi decoding as well as a CRC that corrects up to one bit error. The final probability of error is

$$
\begin{aligned}
& P_{p}\left(\bar{\gamma}_{b i}\right) \\
& =1-\left(\left(1-P_{b}\left(\bar{\gamma}_{b i}\right)\right)^{288}+\left(\begin{array}{c}
288 \\
1
\end{array}\right) P_{b}\left(\bar{\gamma}_{b i}\right)\left(1-P_{b}\left(\bar{\gamma}_{b i}\right)\right)^{287}\right)
\end{aligned}
$$

where $P_{b}\left(\bar{\gamma}_{b i}\right)$ is the probability of error at the output of the the Viterbi decoder which can be determined by equations in [15]. We use the packet error rate computed above as the abstraction for the physical layer (ie. if a packet is received 
correctly/incorrectly over the channel with SINR $\bar{\gamma}_{b i}$ ) in our MAC simulations in the next section.

\section{MAC PRINCIPLES FOR UNDERWATER NETWORKS}

We now apply the above packet level abstraction of the link layer to MAC design and simulation analysis. It has been well documented that the underwater channel conditions, especially the long propagation delay, are detrimental to the performance of traditional MAC techniques. It is important to consider constraints imposed by the link layer hardware on the available choices for MAC design. For example, researchers using commercial off the shelf(COTS) acoustic modems rarely have access to modem firmware that is necessary to implement their custom designed protocols. As such, many previously proposed protocols are somewhat academic in nature, as they make assumptions on PHY layer abilities that are not necessarily tenable with COTS equipment. For example, the MAC protocols of [17] and [18] use handshaking schemes (RTS/CTS) prior to a data transmission. The Micromodem in FH-FSK mode only allows two packet sizes: 21 bits and 32 bytes. If the desired control information cannot be contained in 21 bits then the control packet size itself must be 32 bytes (implying a significant additional 3.2 second transmission delay at $80 \mathrm{bits} / \mathrm{sec}$ and consequent poor channel utilization). Other protocols, such as [19] require node placement information and precise power control. On typical platforms power control may not be available, and, if node positions are not known, accurate underwater localization continues to be a difficult problem.

With the above in mind, we propose a simple ALOHA based scheme with random backoff which makes use of only the simplest link state information. We believe this protocol to be applicable to a wide range of networks implemented on virtually any modem hardware. Previous literature has found that ALOHA based protocols are a good candidate for sparse low data rate networks [20]; the addition of the random backoff mechanism will further assist these networks to cope with (short-term) heavy load bursts such as in a monitoring network employed for event detection. In this case, although long term average load is low, when an event occurs, network load closely approximates saturation conditions.

We next provide the specifics of our proposed MAC protocol, then describe its interactions with the Micromodem FHFSK link layer.

\section{A. ALOHA With Random Backoff}

A state diagram of our proposed MAC protocol is shown in Fig. 8. For backoff purposes, each node uses the parameter, $C W$, the contention window size. When a packet is received for transmission, the node randomly chooses a starting slot value between 0 and $C W-1$. The time duration of each slot, $\sigma$, is an implementation specific parameter which should be long enough to assess channel state information ${ }^{3}$. The node then sets a timer accordingly and begins counting down; the timer will pause whenever the channel is assessed to be busy. When the timer reaches 0 , the node transmits.

\footnotetext{
${ }^{3}$ The backoff time is discretized into slots in order to account for hardware timing constraints and to simplify implementation.
}

The above requires nodes to assess the channel state (busy/idle) continually at each slot boundary. However, many platforms, including the WHOI Micromodem, are not equipped with a separate carrier sense capability. Therefore, each node pauses its timer whenever a packet is acquired for reception at any node. In the case of the Micromodem, all acquired packets are received in their entirety which effectively results in a form of clear channel assessment or carrier sensing. However it is not robust, as evidenced by the case in which an interfering transmission arrives at some time $\tau$ after a packet has been acquired and is being received. The later arrival will not be acquired regardless of its SINR, and (assuming equal packet lengths) there will be a period $\tau$ after the first arrival has been received in its entirety that the channel will be erroneously assessed to be idle.

Our MAC is defined by the two parameters $\sigma$ and $C W$. The slot duration, $\sigma$, must be long enough to assess channel state information. In the micromodem this equals the delay in reporting a matched filter detection. We will tune $C W$ (as a multiple of slot durations) for optimal MAC throughput and verify these dependencies through simulation for selected network environments.

\section{B. ns2 Implementation}

We have developed new MAC, PHY, and propagation models in the popular freeware network simulator, ns-2. Documentation and the code is available at [21]. For each node pair in the network, we develop a power delay profile (PDP) for the specific environmental conditions and geometry using the technique in Section III. This information is used to calculate the SINR at the receiver which in turn specifies the packet error rate for that FH-FSK link; this provides the link layer abstraction in ns-2 for determining whether a transmitted packet is successfully received or lost.

To account for interference on the frequency hopping link layer, we consider signal power components that overlap in time with the reference symbol on a given frequency. For instance, a packet with only one significant arrival in its power delay profile will only interfere if it arrives at the receiver in an interval $\left[m T_{s},(m+1) T_{s}\right)$ before/after the reference packet, where $T_{s}$ is the symbol period. Calculation of the SINR follows from Eq. (14) and finally packets are dropped according to Eq. (15) using the minimum received SINR over the packet duration. This is in contrast to the usual 'collision' assumption whereby all packets are conservatively assumed to be lost if there is any overlap in time.

\section{Simulation of ALOHA with Random Backoff MAC}

For the following simulations, we place a receiving node in the geographic center of the simulation area and randomly deploy traffic generating nodes in a square region around the receiver. We assume a constant packet transmission delay of $3.2 \mathrm{~s}$ and use a slot length of $0.2 \mathrm{~s}$. All throughput estimates are based on averaging of 7 simulation runs (each lasting 30 minutes); approximately 500 packets are transmitted in each run, achieving desired confidence levels. We report normalized throughput by dividing the measured throughput by the link capacity of $1 / 3.2$ packets per second. 


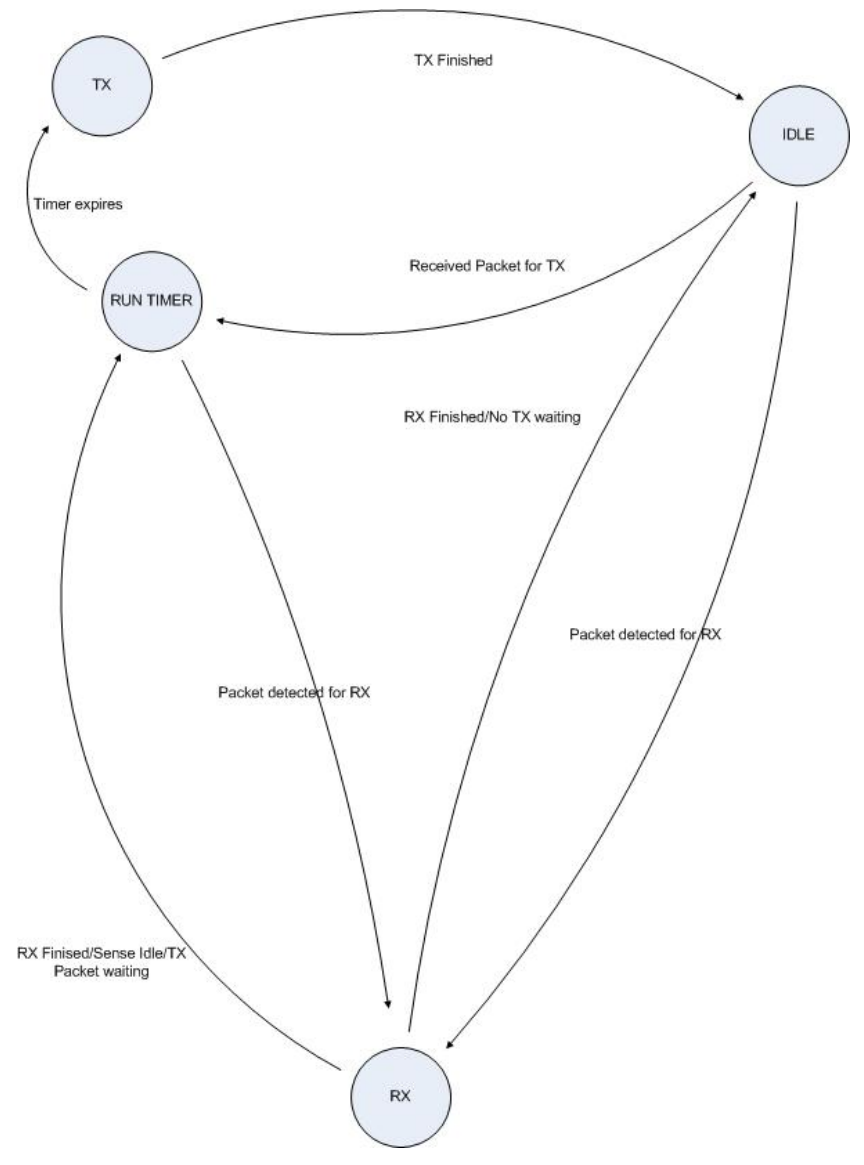

Fig. 8. State transition graph for ALOHA with Random Backoff

In the next set of simulations, the environment SSP 1 shown in Fig. 1 is used with all nodes at 70 meters depth. This deployment is representative of the channel impulse response in the upper panel shown in Fig. 7. In the final simulation we will show the effect that deploying a single node above the thermocline can cause.

Example 1: Saturated Traffic First, simulations are conducted with saturated traffic in order to find the optimum value of $C W$ for 10,15 , and 20 node networks where the nodes are uniformly placed in a square region measuring $500 \mathrm{~m} \times 500 \mathrm{~m}$. The results are given in Fig. 9. We will use these results to tune the $C W$ parameter for further simulations.

Example 2: Poisson Traffic We now present simulation results for 10,15 , and 20 node networks deployed in a $500 \mathrm{~m}$ $\mathrm{x} 500 \mathrm{~m}$ region with Poisson traffic arrivals. $C W$ is set to the value corresponding to peak throughput found in Fig. 9. Specifically we set $C W=48, C W=76$, and $C W=107$ for 10, 15, and 20 nodes respectively. Throughput versus normalized network offered load results are shown in Fig. 10. These results show that the maximum achievable throughput is not dependent on the number of nodes as long as $C W$ is tuned correctly.

Overlaid with the results of our ALOHA with random backoff are simulations carried out with the pure ALOHA protocol. It is important to note that the maximum throughput achieved with the pure ALOHA protocol exceeds the $1 /(2 e)$ theoretical maximum. This is due to the 'collision' model adopted in the simulations that allows the possibility of capture of the

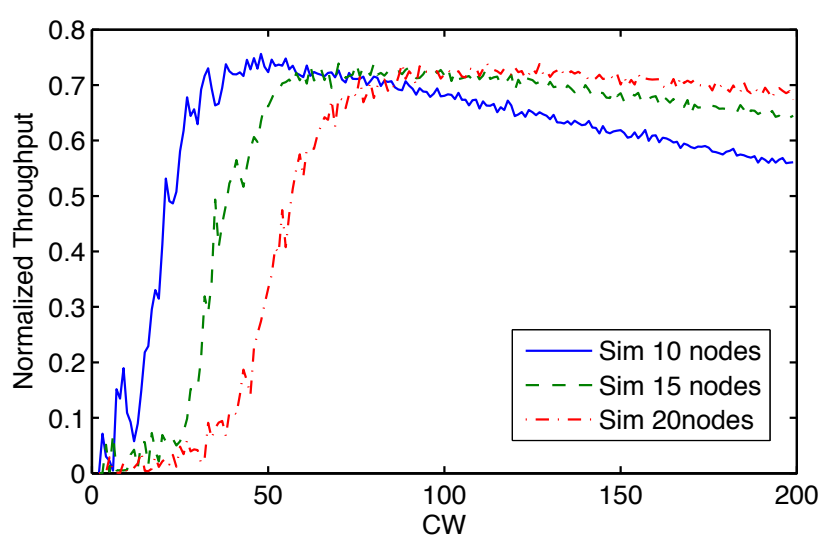

Fig. 9. Throughput vs CW for 10, 15, and 20 nodes with saturated traffic deployed in a $500 \mathrm{~m} \times 500 \mathrm{~m}$ region using the FH PHY.

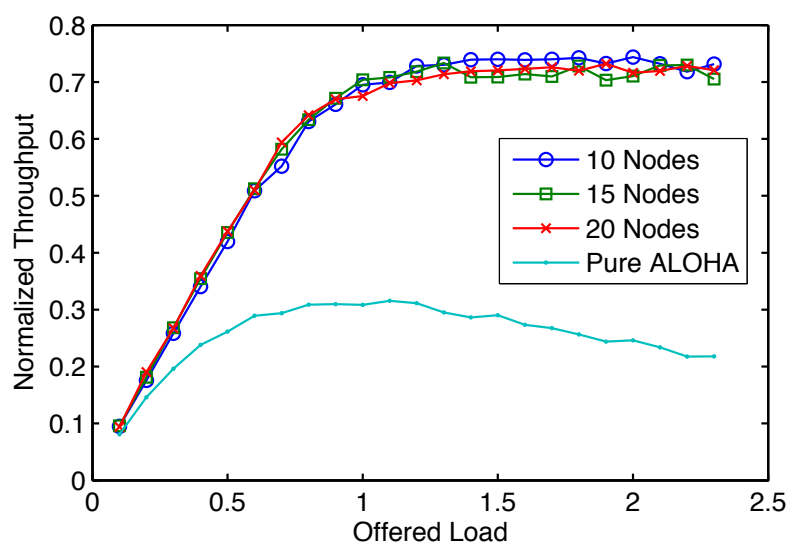

Fig. 10. Throughput vs network offered load in a $500 \mathrm{~m} \times 500 \mathrm{~m}$ region with Poisson traffic for a) 10,15 , and 20 nodes with the ALOHA with random backoff MAC and b) 15 nodes with Pure ALOHA.

stronger packet when two packets overlap. The simulation results nonetheless show that the random backoff mechanism still greatly outperforms the pure ALOHA protocol.

The power expended in underwater sensor networks under moderate to heavy load is generally dominated by the transmit power which is orders of magnitude higher than the power expended in receive or idle modes. As an example, the Micromodem consumes $10 \mathrm{~W}$ while transmitting and only $80 \mathrm{~mW}$ while idle or in receive mode [14]. Given these characteristics, a significant measurement of the energy efficiency of our protocol is the percentage of transmit power wasted in collision which we show vs. the normalized network offered load in Fig. 11. These results show that our protocol offers a significant improvement in energy efficiency over pure ALOHA.

To determine the effect of network size on the achievable throughput we ran simulations of 15 nodes in square regions with dimension measuring $200 \mathrm{~m}, 500 \mathrm{~m}$, and $1 \mathrm{~km}$. The optimal value of $C W$ was found via simulation (using the same method as above) to be 42,76 , and 100 for $200 \mathrm{~m}, 500 \mathrm{~m}$, and $1 \mathrm{~km}$ respectively for $\sigma=0.2 \mathrm{~s}$. The throughput vs. offered load is shown in Fig. 12. As should be expected, the attainable throughput is adversely affected by increased network size. 


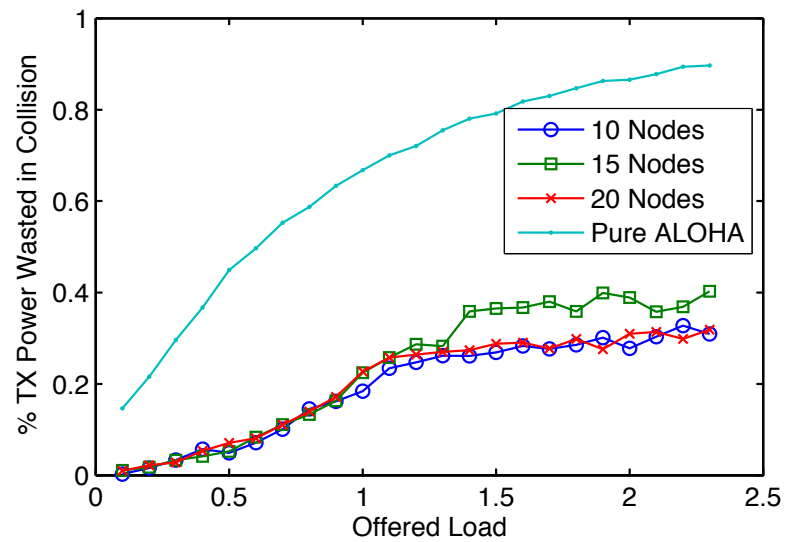

Fig. 11. Percent TX power wasted vs offered load in a 500m x 500m region with Poisson traffic and a) 10, 15, and 20 nodes with ALOHA with random backoff and b) Pure ALOHA with 15 nodes.

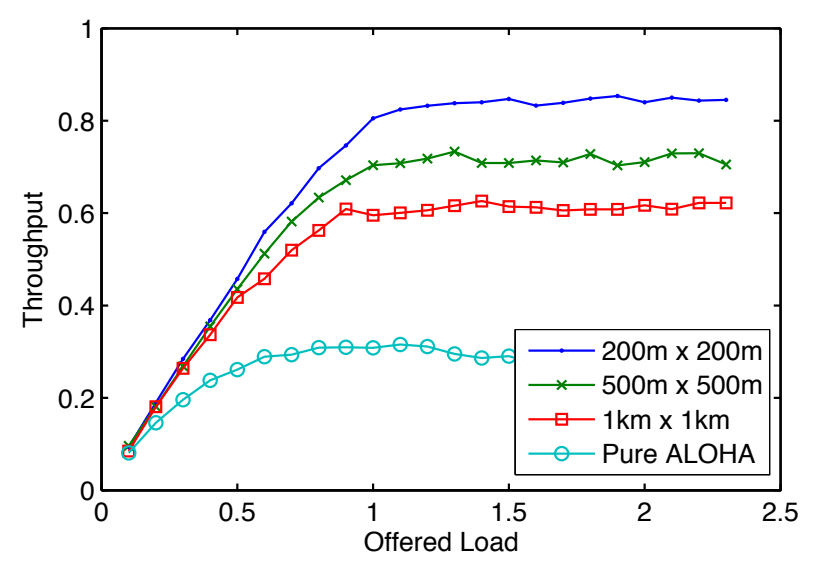

Fig. 12. Throughput vs network offered load for 15 nodes with Poisson traffic a) deployed in $200 \mathrm{~m} \times 200 \mathrm{~m}, 500 \mathrm{~m} \times 500 \mathrm{~m}$, and $1000 \mathrm{~m} \times 1000 \mathrm{~m}$ regions using ALOHA with random backoff and b)500m x 500m region using Pure ALOHA.

\section{Sensitivity Analysis}

In the previous simulation examples, we assumed precise knowledge of the number of nodes and the network area for tuning of the $C W$ parameter. We now present results of simulations where knowledge of network parameters - number of nodes and network size is mismatched with the true values.

If the number of network nodes is incorrectly estimated and $C W$ incorrectly tuned, then the network throughput at heavy loads is negatively impacted relative to that in Fig. 9. To confirm this, we ran simulations of varying numbers of nodes randomly deployed in a $500 \mathrm{~m} \times 500 \mathrm{~m}$ area with $C W$ tuned for a 15 node network. The results for the correctly tuned network and those for the mismatched network are presented in Fig. 13. We found that with $C W$ tuned for $n=15$ nodes $(C W=76)$, throughput at an offered load of 2.5 was within $10 \%$ of the maximum for $6<n<24$.

Error in estimating network dimension (or equivalently, maximum propagation delay) will also result in mismatched $C W$. Fig. 14 shows results of a 15 node network with parameters tuned for $500 \mathrm{~m} \times 500 \mathrm{~m}$ deployment where the actual deployments are $200 \mathrm{~m} \times 200 \mathrm{~m}$ and $1 \mathrm{~km} \times 1 \mathrm{~km}$, respectively. Compared with Fig. 12 with perfect knowledge for $200 \mathrm{~m} \mathrm{x}$

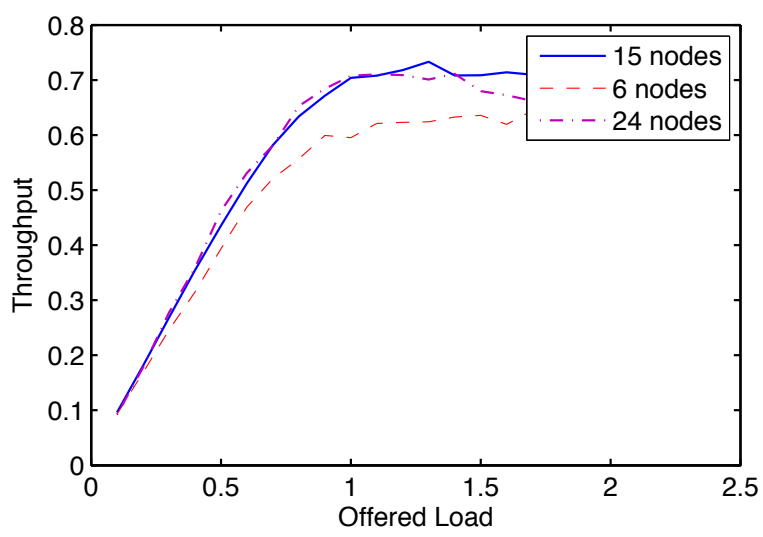

Fig. 13. Throughput vs network offered load for 6,15 , and 24 node networks with saturated traffic deployed in a $500 \mathrm{~m} \times 500 \mathrm{~m}$ region with $\mathrm{CW}$ tuned for 15 nodes.

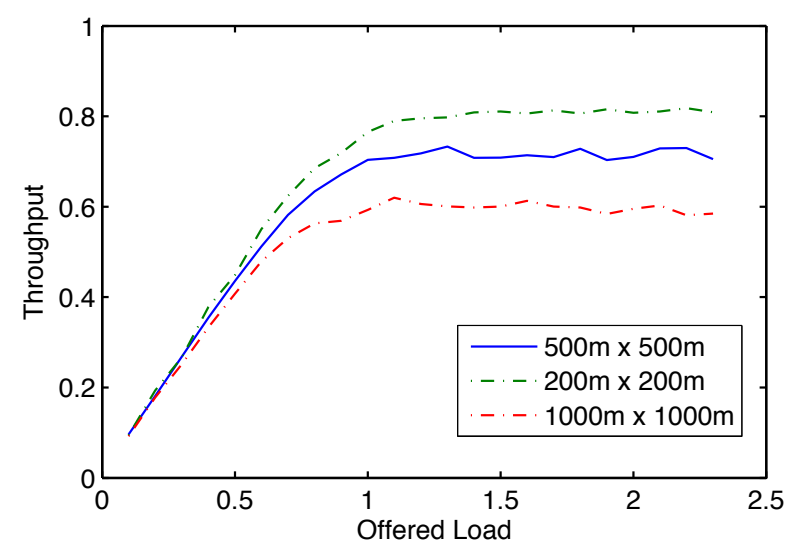

Fig. 14. Throughput vs network offered load for a 15 node network with saturated traffic and deployed in $200 \mathrm{~m} \times 200 \mathrm{~m}, 500 \mathrm{~m} \times 500 \mathrm{~m}$, and $1 \mathrm{~km} \mathrm{x}$ $1 \mathrm{~km}$ region with $\mathrm{CW}$ tuned for a $500 \mathrm{~m} \times 500 \mathrm{~m}$ area.

$200 \mathrm{~m}$ and $1 \mathrm{~km} \times 1 \mathrm{~km}$ network size, the results illustrate that mismatches lead to approximately $4.8 \%$ and $2.5 \%$ degradation in optimum throughput, respectively. This robustness in MAC protocol design suggests that on-line adaptation to network deployment size is feasible.

\section{E. Environmental Effects}

We have shown through simulation that the MAC performance is dependent on the underlying PHY. We have also shown in previous sections the impact of environmental conditions and source and receiver location on link capacity and link performance for the FH-FSK modem. We will now present simulation results that show how node placement within the channel also affects MAC performance and tuning via increased probability of collision. In our previous simulations we placed all nodes, transmitters and receiver, at a depth of $70 \mathrm{~m}$ in SSP 1 . We now compare results of two simulations, the first with the receiver still placed at $70 \mathrm{~m}$ depth and the second with the receiver placed above the thermocline at $10 \mathrm{~m}$ depth. In both simulations transmitting nodes will still be placed at a depth of $70 \mathrm{~m}$. Nodes will be arranged in a star topology with each transmitting node at a range of $1.5 \mathrm{~km}$ from the central receiver. The results of our simulations are shown in Fig. 15. 


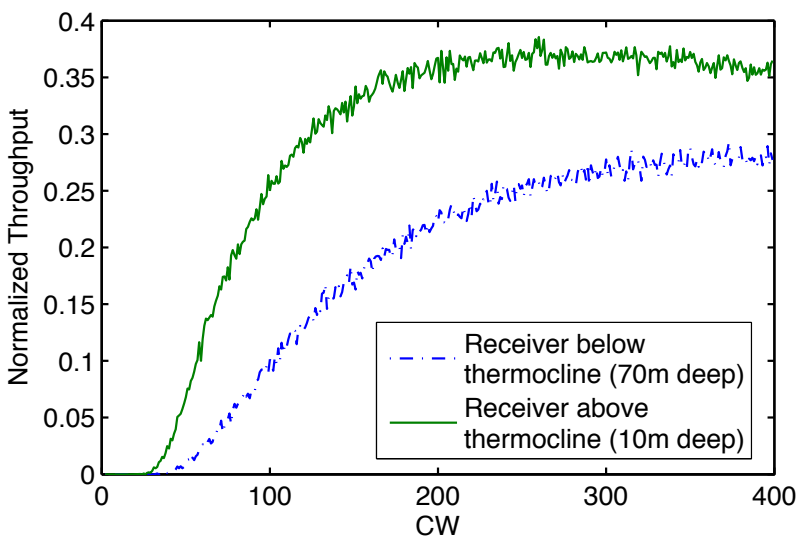

Fig. 15. Throughput vs $C W$ for nodes in a star topology with radius to central receiver of $1.5 \mathrm{~km}$ and a) receiver at depth $70 \mathrm{~m}$ and b) receiver at depth $10 \mathrm{~m}$.

As indicated by Fig. 7, the power delay profile has many more significant arrivals when the receiving node is above the thermocline. This adds both ISI in a single user's transmission, and also increases the chance for interference. This is reflected in the MAC layer by an increased probability of data loss for colliding packets. The end result is reduced optimal throughput and a larger optimal value of $C W$ for the case with the receiver above the thermocline. This clearly shows that MAC optimization in an underwater network is highly dependent upon the specific channel conditions which stem from the environmental effects of the channel.

\section{CONCLUSION}

In this paper, we investigated the influence of environmental conditions and network geometry on several different layers of underwater acoustic communications systems. The capacity computations and FH-FSK link performance show that acoustic conditions and node locations have considerable impact on the maximum achievable bit rate. We then show how acoustic propagation geometry and link level losses affect MAC layer design and tuning for performance optimization.

Our MAC analysis shows that networks using the WHOI Micromodem can increase throughput over pure ALOHA by implementing a backoff rule assuming knowledge of the expected number of contending nodes and the maximum propagation delay. Sensitivity analysis suggests that this is a robust approach; exploring techniques for making this protocol adaptive to network load and channel conditions is left as future work. Despite MAC improvements to a WHOI Micromodem, the achievable rate with current technology is a small fraction of the theoretical channel capacity. This points to the need for continued enhancements to cross layer design approaches that jointly optimize both the link and MAC to achieve the potential of the acoustic channel.

\section{ACKNOWLEDGMENT}

The authors acknowledge the assistance and contributions of Bruce Howe and Matt Grund. Also, the advice of the Guest Editors towards refining this submission is appreciated.

\section{REFERENCES}

[1] "Real-time, long-term ocean and earth studies at the scale of a tectonic plate: Neptune feasibility study," 2000. [Online]. Available: http://www.neptune.washington.edu

[2] M. Grund, L. Freitag, J. Preisig, and K. Ball, "The plusnet underwater communications system: acoustic telemetry for undersea surveillance," IEEE OCEANS 2006, pp. 1-5, Sept. 2006.

[3] M. Chitre, S. Shahabudeen, and M. Stojanovic, "Underwater Acoustic Communications and Networking: Recent Advances and Future Challenges," Marine Technology Science Journal, vol. 42, no. 1, pp. 103-116, Spring 2008.

[4] M. Stojanovic, "On the relationship between capacity and distance in an underwater acoustic communication channel," in WUWNet '06: Proceedings of the 1st ACM international workshop on Underwater networks, 2006, pp. 41-47.

[5] — - "Frequency reuse underwater: capacity of an acoustic cellular network," in WuWNet '07: Proceedings of the second workshop on Underwater networks, 2007, pp. 19-24.

[6] L. Freitag, M. Johnson, M. Grund, S. Singh, and J. Priesig, "Integrated acoustic communication and navigation for multiple uuvs," Proc. IEEE OCEANS 2001, vol. 4, pp. 2064 - 2070, 2001.

[7] M. Porter. (2007) Bellhop gaussian beam/finite element beam code. [Online]. Available: http://oalib.hlsresearch.com/Rays/index.html

[8] M. B. Porter and H. P. Bucker, "Gaussian beam tracing for computing ocean acoustic fields," J. Acoustical Soc. of America, vol. 82, no. 4, pp. 1349-1359, 1987.

[9] R. J. Urick, Principles of Underwater Sound, 3rd ed. New York: McGraw Hill, 1983.

[10] "Apl-uw high-frequency ocean environmental acoustic models handbook," Applied Physics Laboratory, University of Washington, Tech. Rep., October 1994.

[11] R. F. Coates, Underwater Acoustic Systems. New York, NY: John Wiley and Sons, Inc., 1989.

[12] A. Goldsmith, Wireless Communications. New York, NY: Cambridge University Press, 2005.

[13] D. R. Jackson and M. D. Richardson, High-Frequency seafloor Acoustics. New York: Springer, 2007.

[14] L. Freitag, M. Grund, S. Singh, J. Partan, P. Koski, and K. Ball, "The whoi micro-modem: an acoustic communications and navigation system for multiple platforms," Proc IEEE OCEANS 2005, pp. 1086-1092 Vol. $2,2005$.

[15] J. Proakis, Digital Communications, 4th ed. New York: McGraw Hill, 2001.

[16] M. Siderius, M. Porter, P. Hursky, and V. McDonald, "Effects of ocean thermocline variability on noncoherent underwater acoustic communications," J. Acoustical Soc. of America, vol. 121, no. 4, pp. 1895-1908, 2007.

[17] B. Peleato and M. Stojanovic, "A mac protocol for ad-hoc underwater acoustic sensor networks," in WuWNet '06: Proceedings of the 1st workshop on Underwater networks, 2006, pp. 113-115.

[18] V. Rodoplu and M. K. Park, "An energy efficient mac protocol for underwater wireless acoustic networks," Proc. IEEE OCEANS 2005, 2005.

[19] H. Doukkali, L. Nuaymi, and S. Houcke, "Power and distance based mac algorithms for underwater acoustic networks," Proc. IEEE OCEANS 2006, pp. 1-5, 2006.

[20] G. Xie, G. Gibson, and L. Diaz-Gonzalez, "Incorporating realistic acoustic propogation models in simulation of underwater acoustic networks: A statistical approach," Proc. IEEE OCEANS 2006, 2006.

[21] (2007) Fundamentals of networking lab. [Online]. Available: http://ee.washington.edu/research/funlab/uan

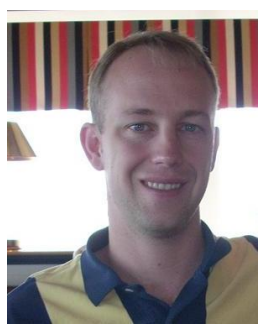

Nathan Parrish is a Ph.D. candidate at the University of Washington. He received his BS degree in electrical engineering from West Point in June of 2001. After graduation, he served as a signal officer in the Army for five years. His current research interests are in ad-hoc networks and underwater acoustic networks. 


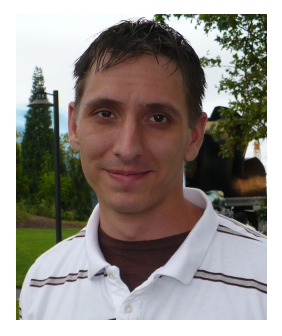

Leonard Tracy is a $\mathrm{PhD}$ student at the University of Washington. He received his BS in Electrical Engineering from the UW in June of 2007. His current research interest is the development of efficient MAC protocols for underwater acoustic networks.

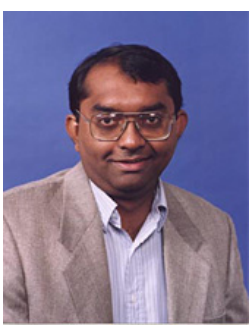

Sumit Roy received the B. Tech. degree from the Indian Institute of Technology (Kanpur) in 1983, and the M. S. and Ph. D. degrees from the University of California (Santa Barbara), all in Electrical Engineering in 1985 and 1988 respectively, as well as an M. A. in Statistics and Applied Probability in 1988. Presently he is Assoc. Chair for Research and Prof. of Electrical Engineering, Univ. of Washington where his research interests include analysis/design of wireless communication and sensor network systems. His recent research emphasis includes wireless LANs (802.11) and wireless MANs (802.16), definition of multi-standard wireless inter-networking and cognitive radios, and sensor networking involving RFID technology. He spent 2001-03 on academic leave at Intel Wireless Technology Lab as a Senior Researcher engaged in systems architecture and standards development for ultra-wideband systems (Wireless PANs) and next generation high-speed wireless LANs; he is currently involved with Intel Labs in enhanced link and MAC layer definitions for Gen2 RFID systems. His activities for the IEEE Communications Society (ComSoc) includes membership of several technical and conference program committees (most recently, Vice Chair of Technical Program Committee for IEEE WCNC05 conference). He has served as Associate Editor for IEEE Trans. Communications and IEEE Trans. on Wireless Communications and currently serves on the Editorial Board for IEEE Trans. Mobile Computing, IEEE Intelligent Transportation Systems and Wiley J. Wireless Communications and Mobile Computing. He was elevated to IEEE Fellow by Communications Society in 2007 for his "contributions to multi-user communications theory and cross-layer design of wireless networking standards".

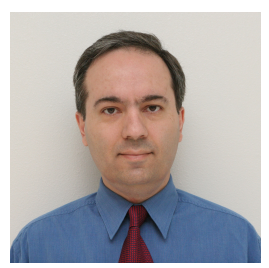

Payman Arabshahi received his M.S. and PhD in Electrical Engineering from the University of Washington in 1994. He is currently a senior research scientist with the University of Washington's Applied Physics Laboratory, and affiliate associate professor of Electrical Engineering at the UW. From 1994-1996 he served on the faculty of the Electrical and Computer Engineering Department at the University of Alabama in Huntsville. From 19972006 he was on the senior technical staff of NASA's Jet Propulsion Laboratory, in the Communications Architectures and Research Section. While at JPL he also served as affiliate graduate faculty at the Department of Electrical Engineering at Caltech, where he taught the three-course graduate sequence on digital communications. He has been a guest editor of the IEEE Transactions on Neural Networks; general co-chair of the 2005 IEEE Swarm Intelligence Symposium, and is currently the Finance Chair of the 2008 ACM Workshop on Underwater Networking. His research interests are in wireless communications and networking, sensor networks, signal processing, data mining and search, and biologically inspired systems.

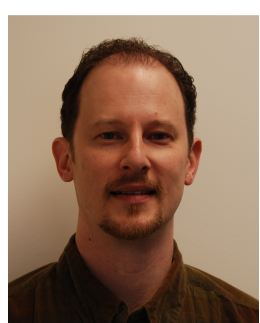

IEEE.
Warren L. J. Fox earned the Ph.D. degree in electrical engineering from the University of Washington, Seattle, WA. He is currently the Director of R\&D and Navy Systems for BlueView Technologies in Seattle, and an Affiliate Associate Professor of Electrical Engineering at the University of Washington. From 1999 to 2007 he was an Electrical Engineer at the Applied Physics Laboratory, University of Washington. From 1996 to 1998 he was a Scientist at the NATO SACLANT Undersea Research Centre in La Spezia, Italy. He is a Senior Member of the 